\title{
Negative induction in instrumental behavior reinforced by central stimulation'
}

\author{
David R. Williams \\ UNIVERSITY OF PENNSYLVANIA
}

\begin{abstract}
Abstraet
When 2 schedules of positive brain stimulation were used in a multiple schedule procedure, a negative induction phenomenon was observed. The effect was strongly time dependent, the depth of the depression was related to the duration of the preceding component, and the effort involved in actual responding did not seem important for the occurrence of the phenomenon. Introduetion
\end{abstract}

In sensory systems the juxtaposition of two stimuli generally increases the apparent difference between them. This "contrast" phenomenon is most often the result of an interaction between excitatory and inhibitory process (e.g., Hartline \& Ratliff, 1958). Pavlov (1927, Ch. XI) pointed out that the "induction" phenomena he observed in classical conditioning were closely analogous to the contrast phenomena of sensory psychology and suggested that both were produced by functionally identical mechanisms. Recently Reynolds (1962) and Catania \& Gill (1964) have reported findings which indicate that induction phenomena occur in instrumental situations as well. ${ }^{2}$ The appearance of such phenomena in instrumental situations is of particular importance because it indicates that a mechanism of interacting excitatory and inhibitory processes is involved in the performance of learned behavior. This paper reports an induction phenomenon in instrumental behavior reinforced by electrical stimulation of the brain. Subjeets

Two female Sherman rats, implanted with monopolar platinumiridium electrodes aimed at the lateral hypothalamic region. Both had been tested for a positive rewarding effect of the electrodes in a lever-pressing situation. Prior to the study R-1 had also been tested in the wheel in an unsystematic fashion; R-2 had never previously been exposed to the wheel apparatus.

Apparatus

A specially constructed low-inertia running wheel $2 \mathrm{ft}$ in diameter was equipped with a generator whose voltage was porportional to speed of running. Running was defined as any speed greater than 1 in/sec.

Procedure

Throughout the experiment, ICS $(0.5$ sec. $60 \mathrm{cps}$ sine wave at $28 \mu$ a) was delivered on a "time schedule" (Williams, in press). Under this procedure, reinforcements are given every time a specified number of seconds of actual running takes place. For example, on a fixed time schedule of $1 \mathrm{sec}$. (FT1), each second of actual running produces a reinforcement; on a variable time schedule of $4.5 \mathrm{sec}$. (VT 4.5), an average of $4.5 \mathrm{sec}$. of actual running is required to produce reinforcement. Time during which running does not take place does not bring $S$ closer to the next reinforcement. Two time schedules were used for each $S$ in a multiple schedule arrangement. For R-1, they were VT 1.5 and VT 4.5. For R-2, they were FT 1 and FT 4 . A tone and a clicker were used to signal which schedule was in force at any time.

At the start of each daily session the high (VT 1.5 or FT 1) and low (VT 4.5 or FT 4) frequency schedules were presented twice, with each presentation lasting $3 \mathrm{~min}$. After this warm-up period, the two schedules were presented for 90 or $270 \mathrm{sec}$., for a total session of $60 \mathrm{~min}$. At the end of $60 \mathrm{~min}$. the component schedule then in force was continued another $30 \mathrm{~min}$. without change. Two balanced sequences of presentation were used. Throughout the session the number of seconds running above criterion speed was recorded in successive $30 \mathrm{sec}$. periods.

\section{Results}

Performance on the multiple schedule procedure appeared to have stabilized after 16 successive daily sessions. Performance on the next four days is presented in Fig. 1, which shows mean number of seconds running per $30 \mathrm{sec}$. recording block. Because the multiple schedule procedure involved a continuous series of alternating components, it was necessary to break the mean curves at some arbitrary place for presentation in the figure. The high frequency component was divided because performance there was relatively invariant.

From inspection of Fig. 1, it is apparent that considerably less running takes place throughout the low frequency component than at any time in the high frequency component. Of marked prominence is the transient depression in running that occurred immediately after introductions of the low frequency component. Virtually no running occurs in the first $30 \mathrm{sec}$. after the change, and performance gradually recovers during the next $60 \mathrm{sec}$. The time-dependence of this induction phenomenon is similar to that reported by Pavlov. A more detailed analysis of the transient depression in running showed that, in the 90 sec. following change to the low frequency component, less running took place if the preceding high frequency component had been in force for 270, rather than $90 \mathrm{sec} .(p<.01$ in both cases, Wilcoxon test).

For the days represented in Fig. 1, the low frequency schedules maintained mean running times of 6.70 and 14.41, and the high frequency schedule times of 17.13 and 23.08, (for $\mathrm{R}-1$ and $\mathrm{R}-2$, respectively) during the

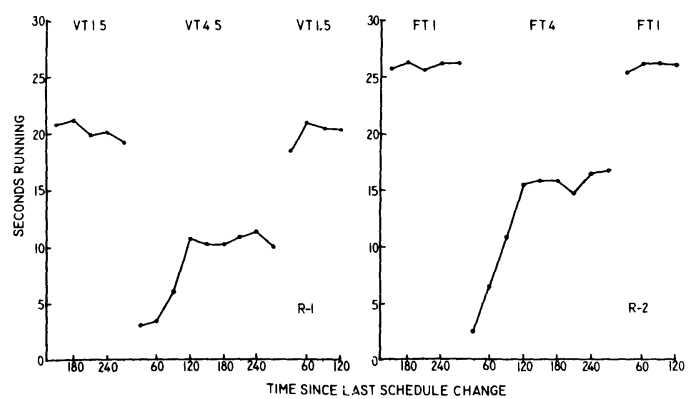

Fig. 1. Mean number of seconds of actual running in successive 30 sec. blocks under both schedules. The high density components have been arbitrarily divided for presentation in the figure. 
30 min. periods of uninterrupted exposure. Thus, although performance under both schedules declined slightly during this period, both schedules were capable of maintaining running for an extended period at approximately the levels reached under the multiple procedure. This finding, and the dependence of depth of depression on the duration of the preceding component, indicate that running under the low frequency schedule is not simply a result of adventitious reinforcement during the multiple procedure.

The contrast phenomenon demonstrated in Fig. 1 emerged only after several sessions. Figure 2 (left) shows the first day of training for R-2. It is evident that performance appropriate to each component carries over into the other, and the net result is a pattern opposite to that demonstrated in Fig. 1. R-1 showed a comparable beginning performance, before gradual development of the results shown in Fig. 1. These data suggest that an abrupt change in frequency of reinforcement is not sufficient to produce the contrast phenomenon; apparently, learning also plays a role in its development.

Following the sessions of Fig。 1, R-2 was placed on a new procedure, to determine whether actual performance of a response (or perhaps the fatigue which might accompany it) was necessary for the contrast phenomenon to occur. Daily sessions were mun as before, but the wheel was locked during the high frequency component. During this time, reinforcements were given in much the same pattern that $\mathrm{S}$ had previously received. As Fig. 2 (right) shows, the contrast phenomenon still took place, even though no responding was possible in the high frequency component. Again, the depression was greater if the high frequency component was in force for $270 \mathrm{sec}$. $(p<.05$, Wilcoxon test). Observation of $S$ gave no suggestion of vigorous activity occurring while the wheel was locked.

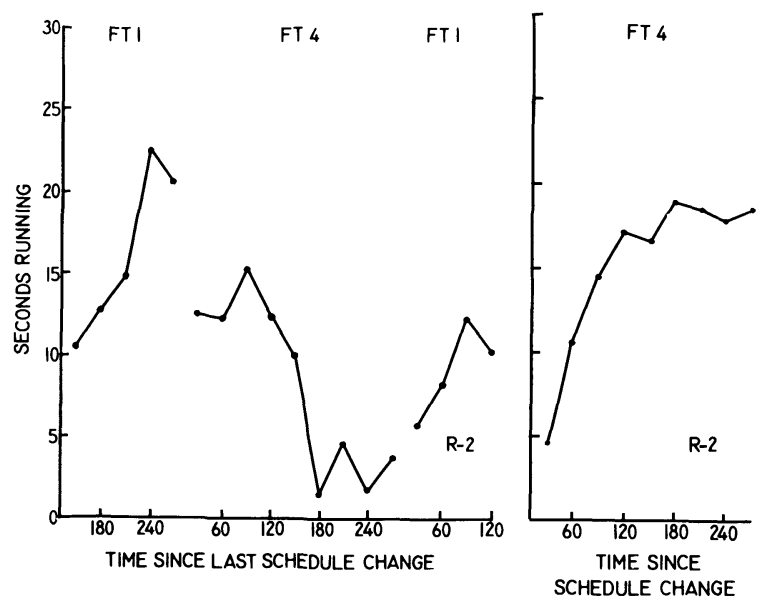

Fig. 2. (Left) Performance on the first day of training, before the induction effect emerged. (Right) Performance in the low density component, when the wheel was locked during the high density component but stimulation was nonetheless delivered.

\section{Diseussion}

Because the low frequency component maintained a substantial level of running, it was possible to demonstrate directly that the high frequency schedule had a depressing effect on subsequent performance. Control procedures indicated that fatigue and adventitious reinforcement were not of major importance to this phenomenon, while the gradual emergence of the phenomenon during training suggests that a shift in the frequency of reinforcement itself is not a sufficient condition. Because the phenomenon is time-dependent, and because it results in an exaggeration of the difference normally maintained by the two schedule conditions, it appears to resemble closely the induction phenomena studied by Pavlov. In Pavlovian terms, these results would be a demonstration of "negative induction"-a transient depression in performance owing to increased inhibition following a sudden reduction in the level of excitation. It may be that the role of training was to condition appropriate levels of excitation to the stimuli signalling each schedule so that, as each was introduced, the corresponding level of excitation was rapidly established. Whether the failure to find the complementary time-dependent parameters of this study or to the use of central stimulation, is currently under investigation.

Appeal to generalized Pavlovian concepts of "excitation" and "inhibition" is futile unless their relationship to external stimuli, and the inductive relationships between them, are carefully specified; further, the relationship between these processes and the performance of instrumental behavior also requires detailed consideration. The present results make several empirical contributions to this general problem. (1) They demonstrate a phenomenon which strongly suggests the operation of an inhibitory process in instrumental behavior. The interaction between the schedule components were related only by generalization. (2) They demonstrate a procedure which, because it produces a strong effect and is readily subject to experimental manipulation, may provide a simple experimental technique for studying Pavlovian mechanisms in instrumental behavior. (3) The use of central stimulation may provide for physiological as well as purely behavior investigation. If the induction effect reported here is related to the locus of stimulation, or if it depends on the integrity of other reward or aversion structures, the phenomenon may provide a useful tool for the physiological investigation of motivational mechanisms in learned behavior.

\section{References}

CATANIA, A. C., \& GILL, C. A. Inhibition and behavioral contrast. Psychon. Sci., 1964, 1, 257-258.

HARTLINE, H. K., \& RATLIFF, F. Spatial summation of inhibitory influences in the eye of Limulus, and the mutual interaction of receptor units. J. gen. Physiol., 1958, 41, 1049-1066.

PAVLOV, I。 P. Conditioned reflexes. Humphrey Milford: Oxford Univ. Press, 1927.

REYNOLDS, G. S. Some limitations on behavioral contrast and induction during successive discrimination. J. exp. Anal. Behav., $1963,6,131,139$.

WILLIAMS, D. R. The relation between response amplitude and reinforcement. J. exp. Psychol., in press.

\section{Notes}

1. This research was supported by a grant, G-23839, from the National Science Foundation.

2. The terms "generalization," "behavioral constrast," and "induction" have been inconsistently used in the literature. Skinner has used "induction" when Pavlov, and most others, use "generalization." Skinner did not devise an opposite term to substitute for Pavlov's original use of "induction"'; Reynolds has introduced the term "behavioral contrast" and has used the term "induction" in the Skinnerian sense. Because of the widespread acceptance of the term "generalization," and because of historical priority, the original Pavolovian usages have been retained here. 